\title{
Study of Nose Leprosy
}

\author{
M. J. OberdoerfFer.
}

It is a well known fact that nose lesions frequently occur in leprosy. It is uncommon in pure maculo-anaesthetic cases, but most of the cutaneous cases show nodules, ulcers, and even a deeply destructive process in the nasal septum and the lower turbinate bones. It has been stated that these lesions most frequently occur in the anterior part of the septum, a matter of special interest in what I shall describe in this paper.

The following gives in tabular form the results of the nose examinations of 160 lepers at Itu, South Nigeria.

Simple ulceration of sept. ant. ...

Plaques or nodules of sept. ant.

Ulcer of sept. post. ... $\quad \ldots$

Ulcer of turb. bone only $\quad \ldots$

Generalised process $\ldots \quad \ldots$

No pathological picture

30

23

3

5

38

61
$18.8 \%$

$14.4 \%$

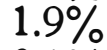

$3.1 \%$

$23.7 \%$

$38.1 \%$

The cases were unselected and of all clinical forms and stages. The predominance of the localisation in the anterior septum is evident. The early plaques and nodules of this localisation can be described as follows:-Thickened, lividlooking, non-inflammatory, submucous nodules, the mucous membrane over which showed no lesion at all. In 5 cases the granuloma formed the centre of 2 to 3 ectatic vessels. The plaques were dry, slightly elevated and livid, surrounded by healthy pink mucosa.

\section{Theories on nose leprosy.}

The frequency of nasal lesions, their common localisation at a very exposed spot, and the tender quality of the nasal mucosa, have given rise to the following theories, each of them doubtless being of some value, whereas none of them can claim to explain all cases of nose leprosy. To mention them and to add a new one with the same limitation, is the task of this paper.

1. The nose lesion as a primary lesion (Sticker). Bacilli enter the mucous membrane through minute erosions and then form granules and ulcers. The modus of infection, comprising the cases, in which leprous material is brought from other leprous lesions of the body, may be :- scratching of the nose (Muir); chronic catarrh, to which in this country the dry harmattan wind might contribute in some cases. 
2. The leprotic process, the result of generalisation in the lymph spaces of the mucous membranes, with consequent localised ulcers (Deycke). It is supposed that infiltration of the lymph spaces with granulomatous tissue manifests itself in a rhinitis sicca (Bluck, Gerber, Sticker, Bengengruen). The last of these supposes that the lymphatic infiltration compresses the minute vessels of the mucous membrane, thus creating ulcers from lack of sufficient blood supply in the periphery. He even thinks of trophoneurotic genesis from the higher sympathetic neuron. That seems improbable, considering the small number of nose lesions form of leprosy.

Deycke is not inclined to accept the primary lesion at all. He thinks the diffuse lymphatic infiltration corresponds with the general onset in the body, succeeded by secondary lesions in the walls of the vessels. The early nose lesions in my material were so circumscribed and abruptly limited by healthy looking mucosa, that I doubt whether this explanation is applicable to all cases.

Sokolowski states very definitely the predominance of the anterior septum as the location of early nose lesions.

Before I dicuss my findings, I want to give some anatomical data of the anterior part of the septum nasi.

The anterior nasal septum has been found to be the most frequent position of nose leprosy. The anterior nasal septum is also the spot in which epistaxis is most common, due to the tender and exposed mucous membrane in this part. Epistaxis is due neither to capillary nor to arterial bleeding. It seems to be a profuse bleeding from temporarily permeable large veins. This theory finds an anatomical basis in the findings of Donogany. The phinologists call this spot of habitual nose bleeding the "Locus Kiesselbachii ". Its histological construction is the following:-The whole septum nasi anterior region is rich in arterial and venous capillaries, which lie in a specific accumulation of glandular tissue these minute vessels have a marked tendency to become ectatic; especially the deeper vessels in the perichondral tissue and in the medium glandular parts show great variation in lumen, and have a remarkably thin wall. The subepithelial vessels are not so liable to ectasis and show more normal conditions, though in old cases of habitual nose bleeding, and, as I have said above, in some of my cases too, the greater subepithlial vessels show ectatic changes, similar to the well known ectasis in the skin vessels of old people.

Donogany supposes that the whole region of the locus Kiesselbachii might be considered as a rudimentary rest of 
the organ of Jacobson, which is still found in earlier forms of vertebrates as a corpus cavernosum-like body.

When we consider the distribution of leprous localisations on the body, we find that the most frequent early lesions occur in parts of the body which show a tendency to blood vessel ectasis, either on the basis of frequent injuries by solar rays or mechanical injuries, or on the basis of the original ectatic structure of the minute vessels. These parts are the ear lobes, the pink cheek, the forearms, etc. (Lewis). The analogy of the distribution of pellagrous lesions has led to theories about a common causative agent in pellagra and leprosy.

Leprosy must until now be considered to be a specific septicaemia with a primary focus, probably in the skin. The frequent symmetrical distribution can only be explained by vascular spread. Is it not probable that the ectatic stasis forms a spot of predilection for the onset of secondary lesions? We found that mechanical or solar influence creates such predilectionary spots, or they may be preformed as developmental rests. I cannot discuss here the analogies between leprosy and pellagra, but I believe that there is a common analogy in that two different disease-producing factors manifest themselves on the same soil-the damaged bloodvessel.

I have stated that the early cases of my material show a remarkably limited submucous appearance. I think that in these cases the histological basis gives more probability of secondary metastatic development than of external origin. The ectatic stasis-producing deep vessels of this region form the basis of haemogenous onset.

\section{Summary.}

The septum anterior nasi, called Locus Kiesselbachii, is doubtless exposed to external lesions, and is thus apt to form a suitable soil for the growth of lepra bacilli, brought to the surface of the mucous membrane in various ways. The histology of this spot gives rise to the opinion that in a certain number of cases the ectatic, abnormal conditions of the deeper vessels form the soil for haemogenous infection.

Acknozeledgments.

I wish to thank Dr. Macdonald, Itu, for his kind permission to perform these studies in his leper colony and to publish the results, and Dr. Muehe, University of Hamburg, for his kind collection of literature on this subject. 